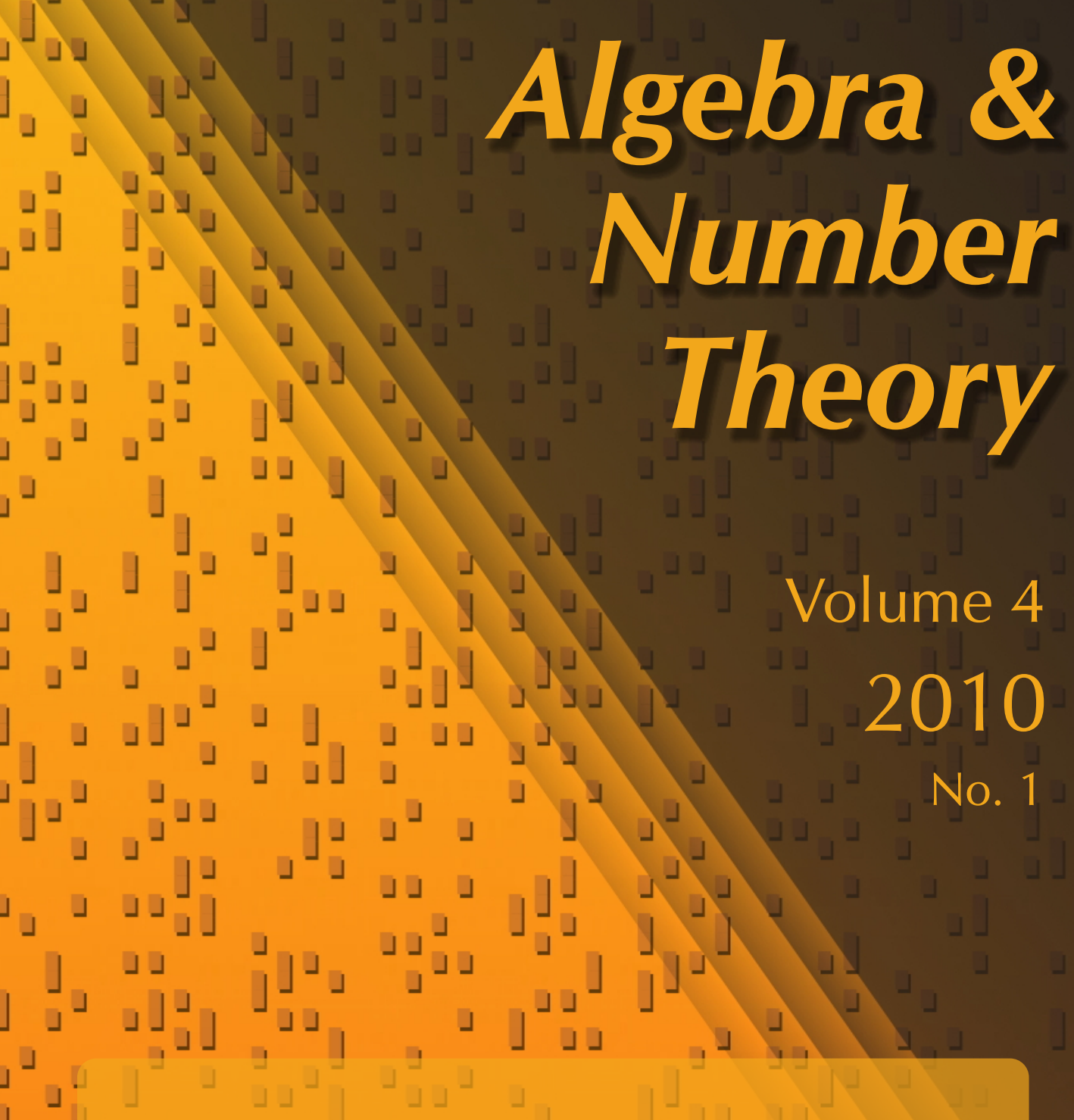

Positive motivic measures are counting measures Jordan S. Ellenberg and Michael Larsen \lrcorner$\lrcorner$.

\lrcorner

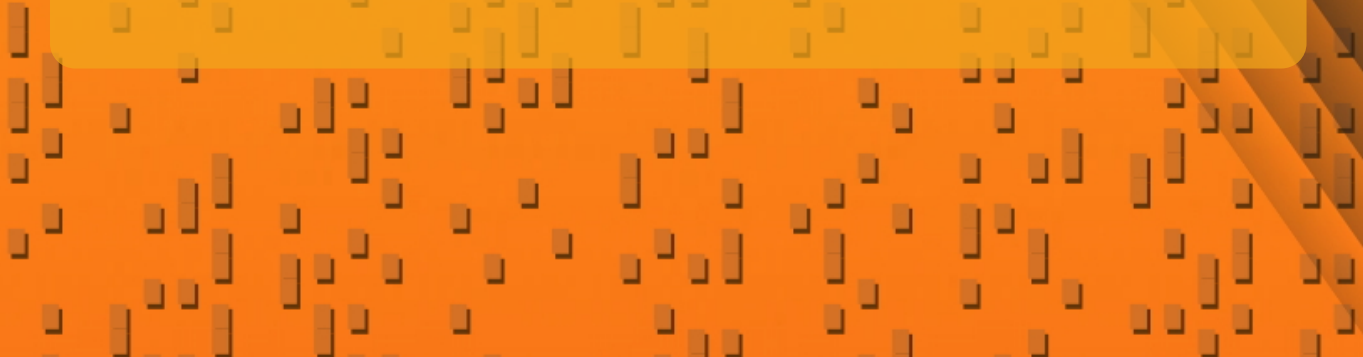

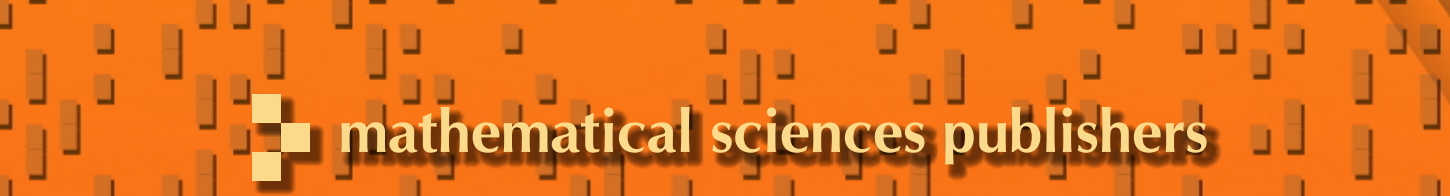




\title{
Positive motivic measures are counting measures
}

\author{
Jordan S. Ellenberg and Michael Larsen
}

\begin{abstract}
A motivic measure is a ring homomorphism from the Grothendieck group of a field $K$ (with multiplication coming from the fiber product over $\operatorname{Spec} K$ ) to some field. We show that if a real-valued motivic measure $\mu$ satisfies $\mu([V]) \geq 0$ for all $K$-varieties $V$, then $\mu$ is a counting measure; that is, there exists a finite field $L$ containing $K$ such that $\mu([V])=|V(L)|$ for all $K$-varieties $V$.
\end{abstract}

Let $K$ be a field. By a $K$-variety, we mean a geometrically reduced, separated scheme of finite type over $K$. Let $K_{0}\left(\operatorname{Var}_{K}\right)$ denote the Grothendieck group of $K$, that is, the free abelian group generated by isomorphism classes [ $V]$ of $K$ varieties, with the scissors relations $[V]=[W]-[V \backslash W]$ whenever $W$ is a closed $K$-subvariety of $V$. There is a unique product on $K_{0}\left(\operatorname{Var}_{K}\right)$ characterized by the relation

$$
[V] \cdot[W]=[V \times W],
$$

where $\times$ denotes the fiber product over Spec $K$. This product gives $K_{0}\left(\operatorname{Var}_{K}\right)$ a commutative ring structure with identity [Spec $K$ ]. For every extension $L$ of $K$, extension of scalars gives a natural ring homomorphism $K_{0}\left(\operatorname{Var}_{K}\right) \rightarrow K_{0}\left(\operatorname{Var}_{L}\right)$. The map $K \mapsto K_{0}\left(\operatorname{Var}_{K}\right)$ can be regarded as a functor from fields to commutative rings. Throughout the paper, we follow the usual convention of writing $\mathbb{L}$ for $\left[\mathbb{A}_{K}^{1}\right]$.

Following the terminology of [Larsen and Lunts 2003], we call a ring homomorphism from $K_{0}\left(\operatorname{Var}_{K}\right)$ to a field $F$ a motivic measure. Note that the original meaning of this term [Hales 2005; Looijenga 2002] is different (though related). If $K$ is a finite field, the map $[V] \mapsto|V(K)|$ extends to a homomorphism $\mu_{K}: K_{0}\left(\operatorname{Var}_{K}\right) \rightarrow \mathbb{Z}$, and therefore to an $F$-valued measure for any field $F$. More generally, if $L$ is an extension of $K$ which is also a finite field, the composition of $\mu_{L}$ with the natural map $K_{0}\left(\operatorname{Var}_{K}\right) \rightarrow K_{0}\left(\operatorname{Var}_{L}\right)$ gives for each $F$ a motivic measure. We will call all such measures counting measures.

In this paper, we consider positive motivic measures, by which we mean $\mathbb{R}$ valued measures $\mu$ such that $\mu([V]) \geq 0$ for all $K$-varieties $V$. We now state our main result.

MSC2000: primary 14F43; secondary $14 \mathrm{G} 15$.

Keywords: motives, motivic measures, finite fields. 
Theorem 1. Every positive motivic measure is a counting measure. In other words, if $K$ is any field and $\mu: K_{0}\left(\operatorname{Var}_{K}\right) \rightarrow \mathbb{R}$ is positive, there exists a finite field $L$ containing $K$ such that $\mu([V])=|V(L)|$ for all $K$-varieties $V$.

Of course, for other choices of $F$ there may still be motivic measures such that $\mu([V])$ lies in some interesting semiring of $F$ for all $K$-varieties $V$. For example, if $F$ is $\mathbb{C}(u, v)$ and $K=\mathbb{C}$, the measure sending $V$ to its Hodge-Deligne polynomial takes values in the semiring of polynomials in $u, v$ whose term of highest total degree is a positive multiple of a power of $u v$.

We begin with a direct proof of the following obvious corollary of Theorem 1 .

Proposition 2. If $K$ is infinite, there are no positive motivic measures on $K_{0}\left(\operatorname{Var}_{K}\right)$.

Proof. Let $\mu$ be such a measure. For any finite subset $S$ of $K$, which we regard as a zero-dimensional subvariety of $\mathbb{A}^{1}$,

$$
0 \leq \mu\left(\mathbb{A}^{1} \backslash S\right)=\mu(\mathbb{L})-|S| .
$$

Thus, $\mu(\mathbb{L}) \geq|S|$ for all subsets $S$ of $K$, which proves the proposition.

For the remainder of the paper we may and do assume that $K$ is finite, of cardinality $q$. We write $\mathbb{F}_{q^{n}}$ for the degree $n$ extension of $K$.

Proposition 3. Let $\Omega^{n}$ denote the variety obtained from $\mathbb{A}^{n}$ by removing all proper affine-linear subspaces defined over $\mathbb{F}_{q}$. Then

$$
\left[\Omega^{n}\right]=(\mathbb{L}-q)\left(\mathbb{L}-q^{2}\right) \cdots\left(\mathbb{L}-q^{n}\right) .
$$

Proof. For any $\mathbb{F}_{q}$-rational affine-linear subspace $A$ of $\mathbb{A}^{n}$, let $A^{\circ}$ denote the open subvariety of $A$ which is the complement of all proper $\mathbb{F}_{q}$-rational affine-linear subspaces of $A$. Then $\left[A^{\circ}\right]=\left[\Omega^{\operatorname{dim} A}\right]$, and one can write recursively

$$
\left[\Omega^{n}\right]=\mathbb{L}^{n}-\sum_{i=1}^{n-1} a_{n, i}\left[\Omega^{i}\right],
$$

where $a_{n, i}$ is the number of $\mathbb{F}_{q}$-rational $i$-dimensional affine linear subspaces of $\mathbb{A}^{n}$. Thus, $\left[\Omega^{n}\right]$ can be expressed as $P_{n}(\mathbb{L})$, where $P_{n} \in \mathbb{Z}[x]$ is monic and of degree $n$. It suffices to prove that $q^{d}$ is a root of $P_{n}(x)$ for all integers $d \in\{1,2, \ldots, n\}$.

For any $d$ in this range $\Omega^{n}\left(\mathbb{F}_{q^{d}}\right)$ is empty. Indeed, if $x \in \mathbb{A}^{n}\left(\mathbb{F}_{q^{d}}\right)$, then the $n$ coordinates of $x$ together with 1 cannot be linearly independent over $\mathbb{F}_{q}$, which implies that $x$ lies in a proper $\mathbb{F}_{q}$-rational affine-linear subspace of $\mathbb{A}^{n}$. Thus,

$$
0=\mu_{\mathbb{F}_{q^{d}}}\left(\Omega^{n}\right)=P_{n}\left(q^{d}\right) .
$$

Corollary 4. If $\mu$ is a positive measure on $K_{0}\left(\operatorname{Var}_{\mathbb{F}_{q}}\right)$, there exists a positive integer $n$ such that $\mu(\mathbb{L})=q^{n}$. 
Proof. If $q^{n-1}<\mu(\mathbb{L})<q^{n}$ for some integer $n$, then $\mu\left(\Omega^{n}\right)<0$, contrary to positivity.

Our goal is then to prove that $\mu(\mathbb{L})=q^{n}$ implies $\mu=\mu_{\mathbb{F}_{q^{n}}}$. We prove first that these measures coincide for varieties of the form Spec $\mathbb{F}_{q^{d}}$ and deduce that they coincide for all affine varieties. As $K_{0}\left(\operatorname{Var}_{\mathbb{F}_{q}}\right)$ is generated by the classes of affine varieties, this implies Theorem 1.

Lemma 5. Let $\mu$ be a real-valued motivic measure of $K_{0}\left(\operatorname{Var}_{\mathbb{F}_{q}}\right)$ and $m$ a positive integer. Then

$$
\mu\left(\operatorname{Spec} \mathbb{F}_{q^{m}}\right) \in\{0, m\} .
$$

If Spec $\mathbb{F}_{q^{m}}$ has measure $m$, then Spec $\mathbb{F}_{q^{d}}$ has measure $d$ whenever $d$ divides $m$.

Proof. As

$$
\mathbb{F}_{q^{m}} \otimes_{\mathbb{F}_{q}} \mathbb{F}_{q^{m}}=\mathbb{F}_{q^{m}}^{m},
$$

the class $x$ of Spec $\mathbb{F}_{q^{m}}$ satisfies $x^{2}=m x$. If $d$ divides $m$,

$$
\mathbb{F}_{q^{d}} \otimes_{\mathbb{F}_{q}} \mathbb{F}_{q^{m}}=\mathbb{F}_{q^{m}}^{d},
$$

so $\mu\left(\operatorname{Spec} \mathbb{F}_{q^{m}}\right)=m$ implies $\mu\left(\operatorname{Spec} \mathbb{F}_{q^{d}}\right)=d$.

Of course,

$$
\mu_{\mathbb{F}_{q^{n}}}\left(\operatorname{Spec} \mathbb{F}_{q^{m}}\right)= \begin{cases}m & \text { if } m \mid n, \\ 0 & \text { otherwise. }\end{cases}
$$

We will prove the same thing for the values of $\mu\left(\operatorname{Spec} \mathbb{F}_{q^{m}}\right)$. We begin with:

Proposition 6. If $\mu(\mathbb{L})=q^{n}$ and $\mu\left(\operatorname{Spec}\left(\mathbb{F}_{q^{k}}\right)\right)=k$ for some $k \geq n$, then

$$
\mu\left(\operatorname{Spec} \mathbb{F}_{q^{m}}\right)= \begin{cases}m & \text { if } m \mid n, \\ 0 & \text { otherwise. }\end{cases}
$$

For any integer $k$, we denote by $X_{k}$ the complement in $\mathbb{A}^{1}$ of the set of all points with residue field contained in $\mathbb{F}_{q^{k}}$.

Proof. By Lemma 5, $\mu$ (Spec $\left.\mathbb{F}_{q^{d}}\right)=d$ when $d$ divides $k$. Choose an $m$ not dividing $k$, and let $Y_{k, m}$ denote the complement in $X_{k}$ of the set of points with residue field $\mathbb{F}_{q^{m}}$. Then

$$
\mu\left(\left[Y_{k, m}\right]\right)=\mu(\mathbb{L})-\sum_{d \mid k} c_{d} d-c_{m} \mu\left(\operatorname{Spec} \mathbb{F}_{q^{m}}\right),
$$

where $c_{i}$ is the number of points in $\mathbb{A}^{1}$ with residue field $\mathbb{F}_{q^{i}}$. From the positivity of $\mu\left(\left[Y_{k, m}\right]\right)$ and the fact that

$$
0=\mu_{\mathbb{F}_{q^{k}}}\left(\left[Y_{k, m}\right]\right)=q^{k}-\sum_{d \mid k} c_{d} d,
$$

we see that $\mu(\mathbb{L})-q^{k}=q^{n}-q^{k}$ must be nonnegative, which is to say $k=n$, and that $\mu\left(\operatorname{Spec} \mathbb{F}_{q^{m}}\right)=0$. 
Proposition 7. If $\mu(\mathbb{L})=q^{n}$, then $\mu\left(\operatorname{Spec} \mathbb{F}_{q^{n}}\right)=n$.

Proof. The assertion is clear for $n=1$, so we assume $n>1$. Let $c_{i}$ denote the number of points in $\mathbb{A}^{1}$ with residue field $\mathbb{F}_{q^{i}}$. Thus $i c_{i} \leq q^{i}-1$ for all $i>1$. If $\mu\left(\operatorname{Spec} \mathbb{F}_{q^{n}}\right)=0$, then $\mu\left(\operatorname{Spec}\left(\mathbb{F}_{q^{i}}\right)\right)=0$ for all $i \geq n$, so for all $k>0$ we have

$$
\mu\left(\left[X_{k}\right]\right) \geq q^{n}-q-\sum_{i=2}^{n-1}\left(q^{i}-1\right) \geq 2 .
$$

Now we consider all curves in $\mathbb{A}^{2}$ of the form $y=P(x)$ where $P(x) \in \mathbb{F}_{q}[x]$ has degree $\leq 2 n$. The total number of such curves is greater than $q^{2 n}$, and for any intersection point $(\alpha, \beta)$ of any two distinct curves of this family, $\alpha$ satisfies a polynomial equation of degree $\leq 2 n$ over $\mathbb{F}_{q}$. Therefore, the open curves

$$
C_{P}:=\left\{(x, P(x)) \mid x \notin \mathbb{F}_{\left.q^{(2 n) !}\right\}},\right.
$$

indexed by polynomials $P$ of degree $\leq 2 n$, each isomorphic to $X_{(2 n) \text { ! }}$, are mutually disjoint. If $C$ denotes the closure of the union of the $C_{P}$ in $\mathbb{A}^{2}$, it follows that

$$
\mu([C])>q^{2 n} \mu\left(\left[X_{(2 n) !}\right]\right)>q^{2 n},
$$

so $\mu\left(\left[\mathbb{A}^{2} \backslash C\right]\right)<0$, which is absurd.

Together, the two preceding propositions imply (1).

We can now prove Theorem 1 . We assume $\mu(\mathbb{L})=q^{n}$. It suffices to check that $\mu([V])=\left|V\left(\mathbb{F}_{q^{n}}\right)\right|$ for all affine $\mathbb{F}_{q}$-varieties $V$.

Each closed point of $V$ with residue field $\mathbb{F}_{q^{d}}$ corresponds to a $d$-element Galois orbit in $V\left(\mathbb{F}_{q^{d}}\right)$. If $d$ divides $n$, it gives a $d$-element subset of $V\left(\mathbb{F}_{q^{n}}\right)$ and the subsets arising from different closed points are mutually disjoint. Since $V\left(\mathbb{F}_{q^{n}}\right)$ is the union of all these subsets, and $\mu\left(\operatorname{Spec} \mathbb{F}_{q^{d}}\right)=d$, we have

$$
\mu([V]) \geq\left|V\left(\mathbb{F}_{q^{n}}\right)\right|
$$

for each $\mathbb{F}_{q}$-variety $V$. However, embedding $V$ as a closed subvariety of $\mathbb{A}^{m}$ for some $m$, the complement $W=\mathbb{A}^{m} \backslash V$ is again a variety, so

$$
\mu([W]) \geq\left|W\left(\mathbb{F}_{q^{n}}\right)\right| .
$$

Since

$$
\begin{aligned}
q^{m n}=\mu\left(\left[\mathbb{A}^{m}\right]\right) & =\mu([V])+\mu([W]) \\
& \geq\left|V\left(\mathbb{F}_{q^{n}}\right)\right|+\left|W\left(\mathbb{F}_{q^{n}}\right)\right| \\
& =\left|\mathbb{A}^{m}\left(\mathbb{F}_{q^{n}}\right)\right|=q^{m n},
\end{aligned}
$$

we must have equality in (2) and (3). 


\section{References}

[Hales 2005] T. C. Hales, "What is motivic measure?", Bull. Amer. Math. Soc. (N.S.) 42:2 (2005), 119-135. MR 2006h:14031 Zbl 1081.14033

[Larsen and Lunts 2003] M. Larsen and V. A. Lunts, "Motivic measures and stable birational geometry”, Mosc. Math. J. 3:1 (2003), 85-95, 259. MR 2005a:14026 Zbl 1056.14015

[Looijenga 2002] E. Looijenga, "Motivic measures", pp. 267-297 in Séminaire Bourbaki, 1999/ 2000, Astérisque 276, Soc. Math. de France, Paris, 2002. MR 2003k:14010 Zbl 0996.14011

Communicated by Bjorn Poonen

Received 2009-07-10 Accepted 2009-08-10

ellenber@math.wisc.edu

Department of Mathematics, University of Wisconsin, 480 Lincoln Drive, Madison, WI 53706, United States http://math.wisc.edu/ ellenber

mjlarsen@indiana.edu

Department of Mathematics, Indiana University,

Bloomington, IN 47405, United States

http://mlarsen.math.indiana.edu/ larsen/ 